\title{
Acute distal achilles tendon rupture reconstruction with the ipsilateral semitendinosus tendon and review of the literature
}

\begin{abstract}
Acute distal Achilles tendon ruptures are uncommon, at the same time these is no standard method of treatment. We propose reconstructing the tendon with the ipsilateral semitendinosus tendon, passed through a transverse tunnel just distal to the region of the Achilles insertion, and weaved through the Achilles tendon, along with 1 or 2 strong sutures. This technique offers a strong fixation, early weightbearing and reduced risk of recurrence by augmenting this degenerated tendon area.
\end{abstract}

Volume 8 Issue 5 - 2017

\author{
Panagiotis G Tsailas \\ Orthopaedic Department, Larisa General Hospital, Greece \\ Correspondence: Tsailas G Panagiotis, Consultant in \\ Orthopaedics, Larissa General Hospital, 6 Mytika Str., Larissa, \\ 4|334, Greece, Tel 307000000000, Email tsailas@gmail.com \\ Received: July 18, 2017| Published: August 23, 2017
}

\section{Introduction}

The Achilles tendon is the largest tendon in the body, it endures a lot of strain and risks rupture. Rupture of the Achilles tendon is most commonly seen at the mid-substance ${ }^{1}$ of the tendon, usually 6 $\mathrm{cm}$ proximally to its insertion to the calcaneus, at an area of reduced vascularity. ${ }^{2}$ Although there is an ongoing debate whether to treat these cases conservatively or surgically, surgical treatment is favored and involves end to end suturing. There are however rare cases of rupture of the distal part of the Achilles which are technically more difficult to repair, since the distal stump is too short or non-existent to hold any sutures. ${ }^{3}$ There have been some reports that use either a pullout technique, or a button technique, or local tendons for the augmentation of the Achilles tendon or, more recently, suture anchors to attach the tendon to the bone.

The aim of this article is to report our technique, which utilizes the ipsilateral semitendinosus tendon as a graft to augment the tendon passed through a transverse trans-osseous tunnel in the calcaneus, just distal to the tendon insertion.

\section{Materials and method}

On November 2013 and on July 2014 two female patient presented in the emergency department of our hospital after an acute failure in plantarflexion of their foot. Both cases had minimal trauma. The first case was a 48 years old woman with a BMI of 29 (overweight) and a history of longstanding pain in the region of the Achilles insertion, the second case was a 57 years old woman with a BMI of 31 (obese) without any history of Achilles pain. No patient has ever received a steroid injection in this area. On examination there was a positive Thomas test and a gap was felt distally in the region of the Achilles tendon insertion. In both cases it was suggested that surgical treatment would be the best option and a written informed consent was obtained. Both cases were operated within $48 \mathrm{~h}$ of injury. Both patients consented to the print and/or electronic publication of the text and any additional components, such as photographs, as well as, an approval by the institution review board was obtained.

\section{Surgical technique}

Prophylactic antibiotics are administered, anaesthesia is attained, a tourniquet is applied to the proximal thigh and the patient is positioned prone on the operating table. The whole leg is prepped and draped distal to the tourniquet, which is inflated after limb exsanguination with a sterile esmach bandage. A traditional paramedian incision, approximately $15 \mathrm{~cm}$ long, medial to the Achilles tendon is performed to avoid injury to the sural nerve. The incision is deepened directly down to the paratenon, which is incised longitudinally to expose the ruptured tendon and bone. We avoid making the flaps superficial to the paratenon so as not to undermining the blood supply to the skin. The distal part of the incision is over the calcaneal tuberosity and full thickness flaps are raised in both directions to the sides of the bone. Once the distal nature of the rupture is verified (Figure 1) the retrieval of the ipsilateral semitendinosus tendon graft is commenced. For the harvest the posterior mini-incision hamstring graft technique described by Prodromos et al. is utilized. ${ }^{4}$ A small, $3 \mathrm{~cm}$ long, incision is made over the semitendinosus on the medial border of the popliteal fossa, the tendon is easily identified and delivered out of the incision (Figure 2a). An index finger follows the tendon proximally and distally. An open striper is first used to free the proximal part of the tendon. Then any fibrous attachments to the tendon, as well as the accessory semitendinosus are easily identified and cut. Finally, with the knee flexed, the index finger follows the tendon to its insertion and where the skin is tented anteriorly a second small, 2 $\mathrm{cm}$, incision is made. The tendon is delivered through the second incision and finally is cut (Figure 2b). The two ends of the tendon graft are prepared by removing any muscle and sutured with No. 2 polyester in an alternating interlocking fashion for $2-3 \mathrm{~cm}$, with at list 5 perforation on each side. The tendon is then subjected to forceful and cyclic loading, its diameter is measured and finally it is stored in moist gauze. The appropriate drill is selected, usually $4.5 \mathrm{~mm}$, and with the help of a drill sleeve, a hole is made just distal to the insertion of the Achilles tendon in the calcaneal tuberosity (Figure 3). With a suture passer one end of the tendon and one or two No. 5 polyester sutures are passed through the drill hole (Figure 4). The tendon stump is cleaned from any degenerated tissue and temporarily approximated to its insertion point with a simple running suture without significantly reducing its length. The two ends of the tendon graft are weaved to each side of the Achilles tendon using a Pulvertaft-like technique (Figure 5). Finally the two sutures ends are tied together. The No. 5 suture is used to suture the Achilles tendon and the graft together, using a Krackow technique, over the whole length of the graft, along each side of the tendon and the two ends are tied proximally (Figure 6). Finally a second No. 5 suture may be used in a modified Kessler technique. The sutures are initially passed through the distal stump. A No. 10 drain is inserted deep to the tendon. The paratenon is then sutured, followed by the subcutaneous tissue and finally the skin. An 
anterior splint is used to immobilize the ankle in 20-25 degrees of plantarflexion (Figure 3).

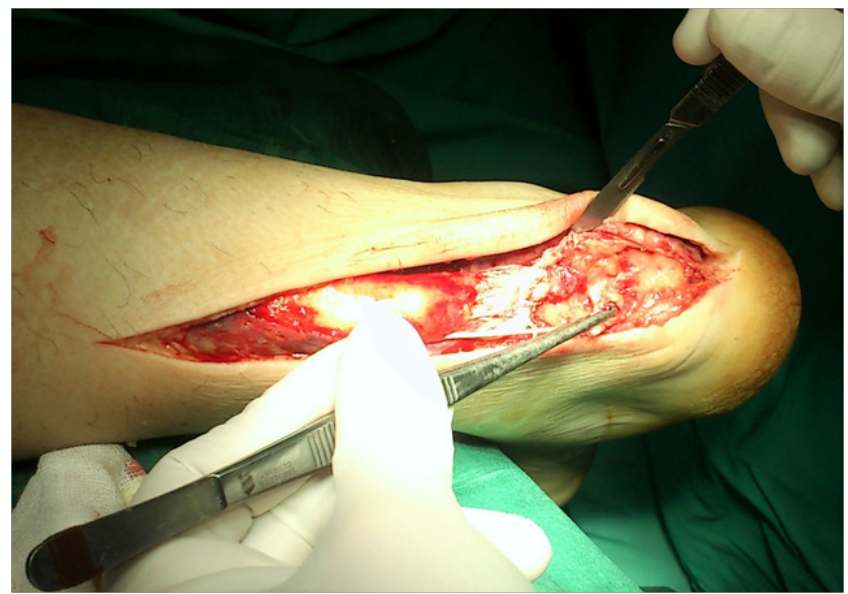

Figure I Distal rupture of Achilles tendon.

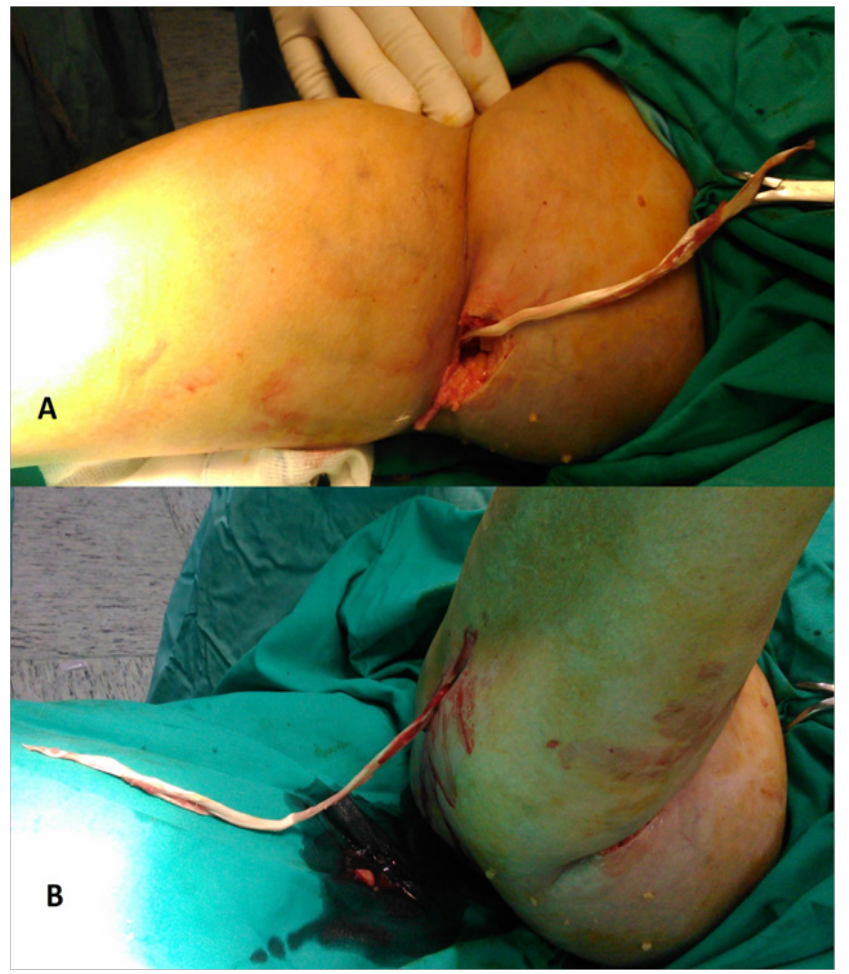

Figure 2A \& B Graft harvesting.

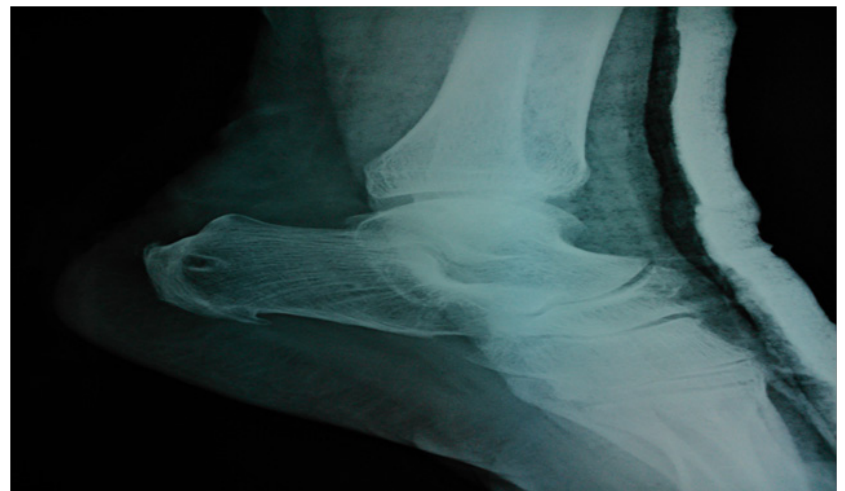

Figure 3 Position of the tunnel on the calcaneus on a lateral radiograph.

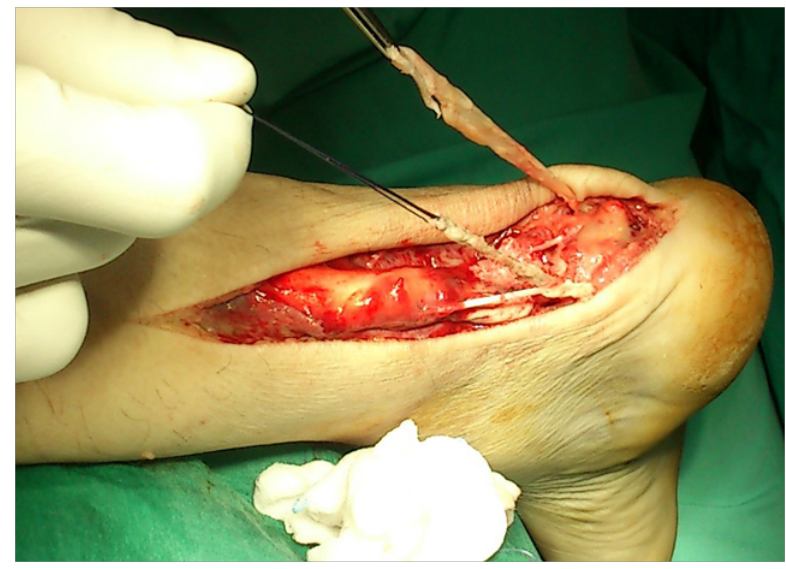

Figure 4 Tendon graft through the bone tunnel.

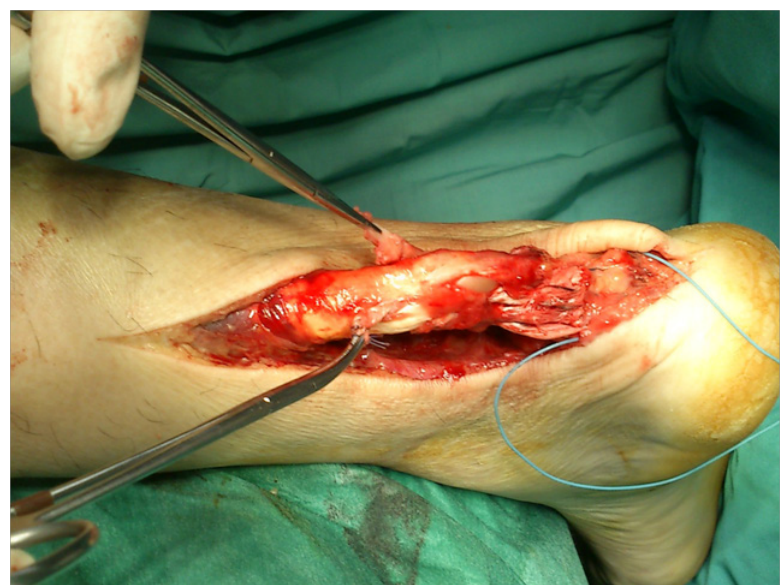

Figure 5 Weaving of the tendon through the Achilles tendon.

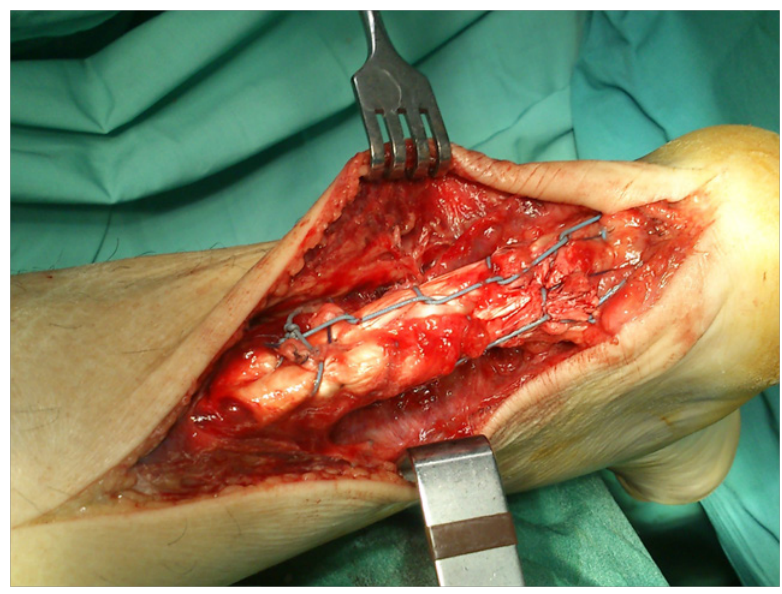

Figure 6 Suturing over the tendons with a \#5 Ethibond suture, passed through the tunnel, using a Krakow technique.

\section{Postoperative management}

The drain is removed on the first or second postoperative day, according to drainage, and the patient is mobilized with 2 crutches without bearing weight on the operated leg. The splint is maintained for two weeks. After 2 weeks the sutures are removed and the splint is replaced by a walking boot with a 20-25 degrees heel insert. Ambulation is progressed to partial weightbearing with 2 crutches. The boot is removed during sleep and for bathing and active range of

Citation: Tsailas PG.Acute distal achilles tendon rupture reconstruction with the ipsilateral semitendinosus tendon and review of the literature. MOJ Orthop Rheumatol. 2017;8(5): I I-12. DOI: 10.15406/mojor.2017.08.00331 
motion exercises are encouraged by the third week. At 1 month the heel insert is replaced by a 10-12 degrees one, and at 6 weeks the heel inserts are removed. At 8 weeks the patient starts using one crutch, at 10 weeks the boot is removed, at 12 weeks the crutch is discontinued.

\section{Results}

In both cases wound healing was complete by 3 to 4 weeks, with the area over the calcaneal tuberosity causing the delay (Figure 7C). There was no deep or superficial infection. There was no morbidity from the graft donor area, and the incisions healed with minimal scaring (Figure 7A,B). The repair was successful and by 10 weeks both patients were weight bearing without any support and continuing with their daily activities and work. There was no ankle stiffness and the range of motion was similar to the normal side (Figure 8AD). There was a bulkiness in the distal part of the Achilles, which however did not pose a significant complaint. Although in one of the patients there is an area of hyposensitivity at the heel she was completely satisfied. Using the modified Mandelbaum and Pavanini scale, reported by Maniscalco et al. ${ }^{5}$ for the clinical assessment of the outcome, both patients had a normal range of motion (10/10) They were completely satisfied with the result, as far as, surgical scar, tolerance to footwear and areas of insensitivity were concerned $(10 / 10)$. The triceps surae strength was similar to normal at 6 months despite the fact that none of them did any dedicated physiotherapy or muscle strengthening (10/10). They had no pain (10/10) and had normal daily activities (10/10). On the basis of this scale both cases had an excellent result (50/50).

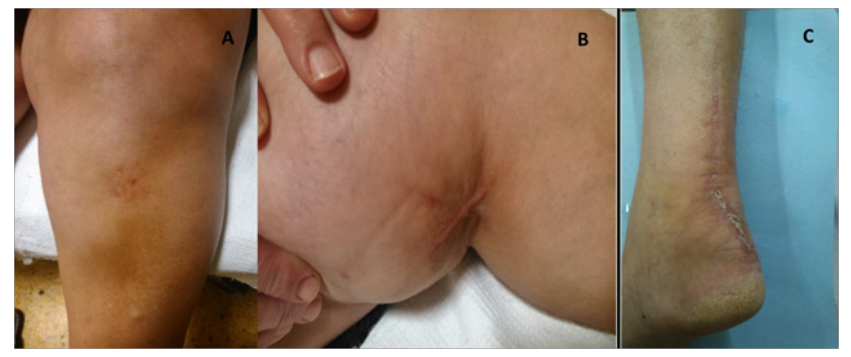

Figure 7A-C Well healed Scars.

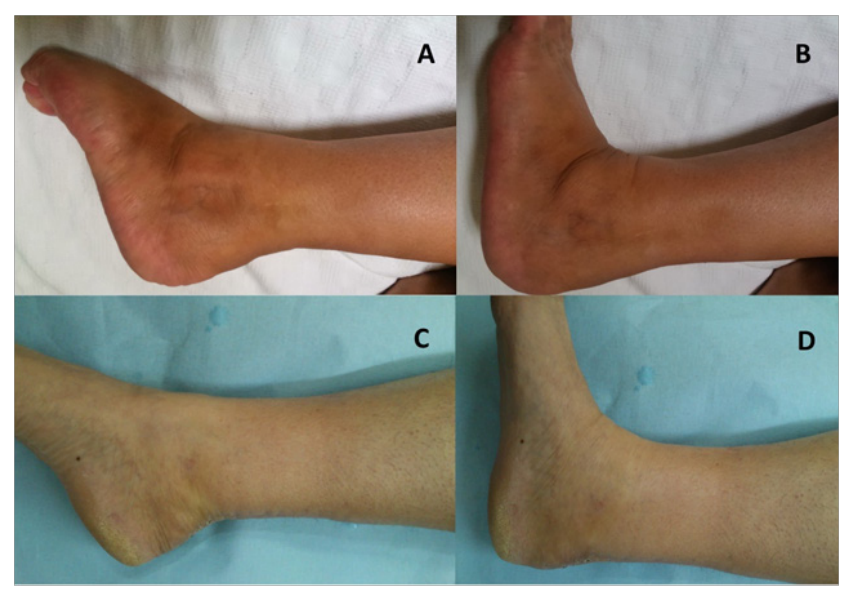

Figure 8A-D Excellent functional result with range of motion similar to the normal side in both cases.

\section{Discussion}

There are three main levels of rupture of the Achilles tendon. Proximal ruptures at the musculotendinus junction, which are common in young individuals, midsection and distal Achilles tendon ruptures which are more common in older individuals, over 40 years of age. ${ }^{5}$ Proximal ruptures are usually treated non-operatively. Midsection ruptures can be treated either non-operatively or operatively, with the surgical treatment giving superior results as far as re-rupture incidence and functional strength is concerned. ${ }^{3}$ The surgical treatment of choice is straightforward and involves end to end repair. Treatment of distal Achilles ruptures is difficult because there is not adequate tendon substance distally for end to end repair, and the tissue is of poor quality due to tendinopathy. ${ }^{6}$ Elderly patients with symptomatic Achilles tendinopathy who develop insertional ruptures are at risk of persistent pain and poor functional outcome. Extensive debridement of the tendinopathy creates a defect that adds to the difficulty of Achilles insertional rupture repair. Fortunately midsection ruptures of the Achilles tendon are more frequent compared to distal insertion site ruptures. At the moment there is no standard method of treatment for acute distal Achilles ruptures. We would like to differentiate avulsion fractures from distal ruptures, because big enough avulsion fractures are treated with osteosynthesis. Although non-operative treatment for distal Achilles tendon ruptures may be applied in low demand patients, surgical treatment is preferred by most authors. The current trend for the treatment of distal ruptures of the Achilles tendon is with the use of suture anchors. In our opinion suture anchors have three downsides. First, there is always the risk of pullout. Second, this is an area of poor vascularity and hence poor degenerated tissue quality for which the anchors do not offer any tissue supplementation, and third, there may be issues with the availability or the cost of such a material especially in poor or developing countries.

Chen et al. ${ }^{7}$ Hanna et al. ${ }^{8}$ Manisculco et al. ${ }^{5}$ Lui et al. ${ }^{1}$ and Kilicoglu et al. ${ }^{9}$ have proposed the use of the Mitek anchor system for the treatment of distal Achilles tendon ruptures, with favorable results. Those authors suggest that this technique offers the advantage of reducing the operative time, of having easy access to the implantation site and of enabling earlier physical therapy. Maniscalco et al. ${ }^{5}$ propose the formation of a trough through the calcaneus cortex for the reattachment of the tendon, which however may shorten the tendon significantly.

Bibbo et al. ${ }^{10}$ reported on the repair of the distal Achilles tendon rupture using a trans-calcaneal pull-out suture technique while Pavlou et al. ${ }^{11}$ combined the trans-calcaneal suture technique of Bibbo ${ }^{10}$ with the suture anchor technique of Lui. ${ }^{1}$ Fanter et al. ${ }^{12}$ in a laboratory study suggested the use of a trans-calcaneal button type technology toward the plantar surface of the foot. The trans-calcaneal drill pin passage appeared to be anatomically safe, and the use of suture button technology with a Krackow stitch for Achilles tendon insertional repair significantly increased repair strength.

There have been several reports of tendon transfers for the treatment of Achilles tendon rupture and tendinosis. One of the most commonly transferred tendons has been the flexor hallucis longus. Wong and $\mathrm{Ng}^{6}$ used a Modified flexor hallucis longus transfer for Achilles insertional rupture in elderly patients. Hahn et al. ${ }^{13}$ also reported on the treatment of chronic achilles tendinopathy and ruptures with flexor hallucis tendon transfer. Lui ${ }^{14}$ reported a minimally invasive flexor hallucis longus transfer for the management of acute achilles tendon rupture associated with tendinosis. The flexor hallucis tendon transfer has also been used for the treatment of chronic irreparable Achilles tendon ruptures by Rahm et al. ${ }^{15}$ The use of the flexor hallucis tendon transfer has been associated with a trend toward reduction in peak pressure loading on the distal phalanx using pedobarography by Coull et al. ${ }^{16}$ and Richardson et al. ${ }^{17}$ showed decreased flexion power at the interphalangeal joint as demonstrated by decreased distal phalangeal pressure $(\mathrm{p}<0.05)$. However, these appear to be laboratory findings, 
as patient function remains high during activities of daily living. A technique using a section of the flexor digitorum longus to repair chronic ruptures was reported by Mann et al. ${ }^{18}$. The peroneus brevis tendon has also been commonly used as a tendon graft for the Achilles tendon rupture. ${ }^{19-22}$ Although a mild objective eversion weakness was reported no objective plantarflexion weakness was found after the use of the peroneus brevis tendon transfer and subjective assessment reveals no functional compromise. ${ }^{23}$ Sebastian et al. ${ }^{24}$ examined the in vitro mechanical properties of a reconstructed Achilles tendon with the peroneus brevis or the flexor hallucis longus tendons in a human cadaver model and found that the mean failure load was significantly higher in the peroneus brevis group. However, the greater failure loads observed with the use of peroneus brevis may not be clinically relevant, because of the magnitude of the peak loads observed in the cadaveric model. The use of the plantaris tendon has been suggested for the treatment of rupture of the Achilles tendon as autogenous suturing material by Dekker \& Bender. ${ }^{25}$ However the plantaris tendon is very inconsistent, as far as its diameter is considered, which is usually very thin, and there are cases when it might be completely absent, in as much as $7-20 \%$ of the limbs. ${ }^{26}$

Free gracilis and semitendinosus tendon graft have only been described in the reconstruction of chronic ruptures with large tendon defects. ${ }^{27-30}$ Besse et al. ${ }^{31}$ used tendon grafts with bone blocks from the knee extensor mechanism and reported satisfactory results with their technique.

During our review of the literature we discovered that our technique is somewhat similar to the one described by $\mathrm{Ji}$ et al. ${ }^{29}$ for the augmentation of a large defect with semitendinosus tendon graft, as far as the calcaneal tunnel is concerned. However in their case the tendon graft is used in a figure of eight pattern between the bone and the tendon and it is not weaved to the tendon as in our case. We believe that our technique offers better tendon fixation and strength. Although there is no biomechanical study to support such an allegation, in their report one of the two presented patients suffered several occasions of re-rupture. In their operative technique they also state that they use a four strand suture anchor into the calcaneal tuberosity so as to maximize fixation which we do not use.

Our rehabilitation protocol seems to be simpler and faster compared to most of the rehabilitation protocols with suture anchor techniques. A somewhat similar rehabilitation program is followed by Lui et al. ${ }^{14}$ with gradual weight bearing walking starting on the second week with the aim of full weight bearing on the seventh postoperative week, and gradual ankle mobilization exercise starting on the second postoperative week. Maniscalco et al. ${ }^{5}$ have a slower program, beginning weight bearing coupled with range-of-motion exercises one month post-surgery, with a hinged walking boot that permits full plantarflexion but blocks dorsiflexion at $10^{\circ}$ degrees for 8 weeks. According to Kilicoglu et al. patients are allowed to walk non-weightbearing in a cast with two crutches for 4 weeks, and then partial weight bearing in a below knee cast; progressing to full bearing at 10 weeks with a heel support below the cast. At 10 weeks, the cast is removed, and prescribed a hinged ankle foot orthotics (HAFO) limiting dorsiflexion. Double heel raises progressing to single heel raises are begun as tolerated by the patient. This very protective rehabilitation protocol is probably the reason for their significantly less mean dorsiflexion of the injured side than that of the normal side, as well as significant decrease in ankle joint plantarflexion power.

\section{Conclusion}

Reconstruction of the distal Achilles tendon rupture with an ipsilateral semitendinosus tendon graft offers ease of harvest without any donor site morbidity, is strong enough and along with our weaving technique allows early active range of motion and partial weight bearing and acts as a biological scaffold that augments the degenerated Achilles tendon.

\section{Acknowledgments}

None.

\section{Conflicts of interest}

None.

\section{References}

1. Lui TH. Fixation of tendo Achilles avulsion fracture. Foot Ankle Surg. 2009;15(2):58-61.

2. Lin JL. Tendon transfers for Achilles reconstruction. Foot Ankle Clin. 2009;14(4):729-744.

3. Wilkins R, Bisson LJ. Operative versus nonoperative management of acute Achilles tendon ruptures: a quantitative systematic review of randomized controlled trials. Am J Sports Med. 2012;40(9):2154-2160.

4. Prodromos CC, Han YS, Keller BL, et al. Posterior mini-incision technique for hamstring anterior cruciate ligament reconstruction graft harvest. Arthroscopy. 2005;21(2):130-137.

5. Maniscalco P, Bertone C, Bonci E, et al. Titanium anchors for the repair of distal Achilles tendon ruptures: preliminary report of a new surgical technique. J Foot Ankle Surg. 1998;37(2):96-100.

6. Wong MW, Ng VW. Modified flexor hallucis longus transfer for Achilles insertional rupture in elderly patients. Clin Orthop Relat Res. 2005;(431):201-206.

7. Chen D, Wertheimer S. A new method of repair for rupture of the Achilles tendon. J. Foot Surg. 1992;31(5):440-445.

8. Hanna JR, Russell RD, Giacopelli JA. Repair of distal tendo Achillis rupture with the use of the Mitek Anchor System.J Am Podiatr Med Assoc. 1993;83(12):663-668.

9. Kiliçoğlu O, Türker M, Yildız F, et al. Suture anchor tenodesis in repair of distal Achilles tendon injuries. Eur J Orthop Surg Traumatol. 2014;24(1):117-122.

10. Bibbo C, Anderson RB, Davis WH, et al. Repair of the Achilles tendon sleeve avulsion: quantitative and functional evaluation of a transcalcaneal suture technique. Foot Ankle Int. 2003;24(7):539-544.

11. Pavlou G, Roach R, Salehi-Bird S. Repair of the achilles tendon sleeve avulsion: a transcalcaneal suture technique.Foot Ankle Int. 2009;30(1):65-67.

12. Fanter NJ, Davis EW, Baker CL. Fixation of the Achilles tendon insertion using suture button technology. $A m J$ Sports Med. 2012;40(9):2085-2091.

13. Hahn F, Meyer P, Maiwald C, et al. Treatment of chronic achilles tendinopathy and ruptures with flexor hallucis tendon transfer: clinical outcofme and MRI findings. Foot Ankle Int. 2008;29(8):794-802.

14. Lui TH. Minimally invasive flexor hallucis longus transfer in management of acute achilles tendon rupture associated with tendinosis: a case report. Foot Ankle Spec. 2012;5(2):111-114.

15. Rahm S, Spross C, Gerber F, et al. Operative treatment of chronic irreparable Achilles tendon ruptures with large flexor hallucis longus tendon transfers. Foot Ankle Int. 2013;34(8):1100-1110.

16. Coull R, Flavin R, Stephens MM. Flexor hallucis longus tendon transfer: evaluation of postoperative morbidity. Foot Ankle Int. 2003;24(12):931-934

17. Richardson DR, Willers J, Cohen BE, et al. Evaluation of the hallux morbidity of single-incision flexor hallucis longus tendon transfer. Foot Ankle Int. 2009;30(7):627-630. 
18. Mann RA, Holmes GB, Seale KS, et al. Chronic rupture of the Achilles tendon: a new technique of repair. J Bone Joint Surg Am. 1991;73(2):214-219.

19. Pérez Teuffer A. Traumatic rupture of the Achilles Tendon. Reconstruction by transplant and graft using the lateral peroneus brevis. Orthop Clin North Am. 1974;5(1):89-93.

20. Turco V, Spinella AJ. Peroneus brevis transfer for Achilles tendon rupture in athletes. Orthop Rev. 1988;17(8):822-824,827-828.

21. Turco VJ, Spinella AJ. Achilles tendon ruptures-peroneus brevis transfer. Foot Ankle. 1987;7(4):253-259.

22. Singh A, Nag K, Roy SP, et al. Repair of Achilles tendon ruptures with peroneus brevis tendon augmentation. J Orthop Surg (Hong Kong). 2014;22(1):52-55.

23. Gallant GG, Massie C, Turco VJ. Assessment of eversion and plantar flexion strength after repair of Achilles tendon rupture using peroneus brevis tendon transfer. Am J Orthop (Belle Mead NJ). 1995;24(3):257-261.

24. Sebastian H, Datta B, Maffulli N, et al. Mechanical properties of reconstructed achilles tendon with transfer of peroneus brevis or flexor hallucis longus tendon. J Foot Ankle Surg. 200746(6):424-428.
25. Dekker M, Bender J. Results of surgical treatment of rupture of the Achilles tendon with use of the plantaris tendon. Arch Chir Neerl. 1977;29(1):39-46

26. Simpson SL, Hertzog MS, Barja RH. The plantaris tendon graft: an ultrasound study. J Hand Surg [Am]. 1991;16:708-711.

27. Maffulli N, Spiezia F, Testa V, et al. Free gracilis tendon graft for reconstruction of chronic tears of the Achilles tendon. J Bone Joint Surg Am. 2012;94(10):906-910.

28. Sarzaeem MM, Lemraski MM, Safdari F. Chronic Achilles tendon rupture reconstruction using a free semitendinosus tendon graft transfer. Knee Surg Sports Traumatol Arthrosc. 2012;20(7):1386-1391.

29. Ji JH, Kim WY, Kim YY, et al. Semitendinosus tendon augmentation for a large defect after Achilles tendon rupture: two case reports. Foot Ankle Int. 2007;28(10):1100-1103.

30. Dumbre Patil SS, Dumbre Patil VS, Basa VR, et al. Semitendinosus Tendon Autograft for Reconstruction of Large Defects in Chronic Achilles Tendon Ruptures. Foot Ankle Int. 2014;35(7):699-705.

31. Besse JL, Lerat JL, Moyen B, et al. Achilles tendon repair using a bonetendon graft harvested from the knee extensor system: three cases. $J$ Foot Ankle Surg. 1999;38(1):70-74. 